\title{
Vaskuläre Läsionen des Skeletts - Pathologie und Genetik
}

\section{Einleitung}

Vaskuläre Läsionen sind häufig und treten z. B. in Form vorwiegend oberflächlicher infantiler Hämangiome bei ca. $4 \%$ aller $\mathrm{Neu}$ geborenen auf. Auch in der orthopädischen Praxis sind Gefäßanomalien präsent: Skelettale vaskuläre Läsionen werden bei ca. $10 \%$ aller Menschen beschrieben, und betreffen meist das Achsenskelett in Form von venösen Malformationen (früher sogenanntes „Hämangiom“ des Knochens).

Das Spektrum gefäßassoziierter Veränderungen ist breit und reicht von gutartigen umschriebenen indolenten Lokalbefunden bis zu generalisiertem Befall mit infauster Prognose. Für eine adäquate Einordnung und erfolgreiche Therapie ist eine einheitliche Klassifikation von entscheidender Bedeutung. Die Entdeckung neuer molekularer Grundlagen hat in den letzten zwei Jahrzehnten wesentlich dazu beigetragen, eine valide Klassifikation zu entwickeln.

Vaskuläre Läsionen können isoliert sporadisch oder seltener familiär, einem be- stimmten Erbgang folgend (ca. $2 \%$ ) auftreten. Die klinische Ausprägung ist durchaus sehr variabel auch innerhalb einer Familie, sie kann symptomlos verlaufen, mit einer Progredienz (kontinuierliche Größenzunahme) oder durch exogene Einflüsse (z. B. Infektionen, hormonelle Dysbalancen, Thrombosierungen) mit sekundären Symptomen und Komplikationen verbunden sein (Eivazi 2014). Eine Besonderheit stellen die syndromalen Entwicklungsstörungen mit assoziierten vaskulären Veränderungen dar, wie das PTEN- Hamartom-Tumor-Syndrom (autosomal dominanter Erbgang) oder das ProteusSyndrom (somatische Mutation im AKT1 Gen, sporadische Genese, siehe unten). Der Zusammenhang mit den vaskulären Malformationen ist noch nicht vollständig geklärt (Duffy 2010).

\section{Klassifikation vaskulärer Läsionen}

Historische beschreibende Klassifikationen werden seit 1996 von einer biologisch orientierten Klassifikation der International
Society for the Study of Vascular Anomalies (ISSVA) abgelöst. In diesem Klassifikationssystem werden vaskuläre Malformationen als strukturelle Anomalien von proliferativen vaskulären Tumoren unterschieden (siehe > Tab. 1 sowie Mulliken 2013). Die ISSVA Systematik wird allmählich auch in die WHO-Klassifikation integriert und lässt sich ebenfalls auf vaskuläre Läsionen des Skeletts anwenden (Bruder et al. 2009).

\section{Vaskuläre Malformationen}

Das Konzept vaskulärer Malformationen geht von strukturellen Fehlern in der embryonalen Morphogenese während der 4.10. Schwangerschaftswoche aus. Vaskuläre Malformationen manifestieren sich eher bei jüngeren Patienten und sind meist oberflächlich lokalisiert. Die Einteilung der Gefäßmalformationen richtet sich nach den Gefäßkomponenten. Dementsprechend lassen sich kapilläre von lymphatischen, venösen und arteriovenösen Malformationen unterscheiden. Gemischte Malformationen mit mehreren Komponenten kommen vor. Der morphologische Wandaufbau sollte 
\Tab. 1 Vaskuläre Läsionen

\begin{tabular}{|c|c|c|c|c|}
\hline \multicolumn{3}{|l|}{ Vaskuläre Tumoren } & \multicolumn{2}{|l|}{ Vaskuläre Malformationen } \\
\hline Gutartig & Lokal Aggressiv & Maligne & Einfach & Kombiniert \\
\hline Infantiles Hämangiom & Kaposiformes Hämangioendotheliom & Angiosarkom & Kapilläre Malformation (K) & KVM, KLM \\
\hline Kongenitales Hämangiom & Retiformes Hämangioendotheliom & $\begin{array}{l}\text { Epitheloides Häman- } \\
\text { gioendotheliom }\end{array}$ & Lymphatische Malformation (LM) & LVM, KLVM \\
\hline Epitheloides Hämangiom & Pseudomyogenes Hämangioendotheliom & & Venöse Malformation (VM) & \\
\hline ‘Tufted’ Hämangiom & PILA+, Dabska§ Tumor & & Arteriovenöse Malformation (AVM) & KLAVM \\
\hline Spindelzell-Hämangiom* & Composit Hämangioendotheliom & & Arteriovenöse Fistel & \\
\hline Pyogenes Granulom & Kaposi Sarkom & & & \\
\hline
\end{tabular}

Gekürzte und modifizierte ISSVA Klassifikation für vaskuläre Läsionen der International Society for the Study of Vascular Anomalies

* von den meisten Autoren heute als Malformation angesehen

+ papilläres intralymphatisches Angioendotheliom (Fanburg-Smith J et al. Am J Surg Pathol 23: 104; 1999)

$\S$ malignant endovascular papillary angioendothelioma (Dabska M, Cancer 24: 503; 1969)

fett $=$ auch als Primärläsion im Knochen beschrieben

fett kursiv = bisher nur Einzelfälle primär im Knochen beschrieben
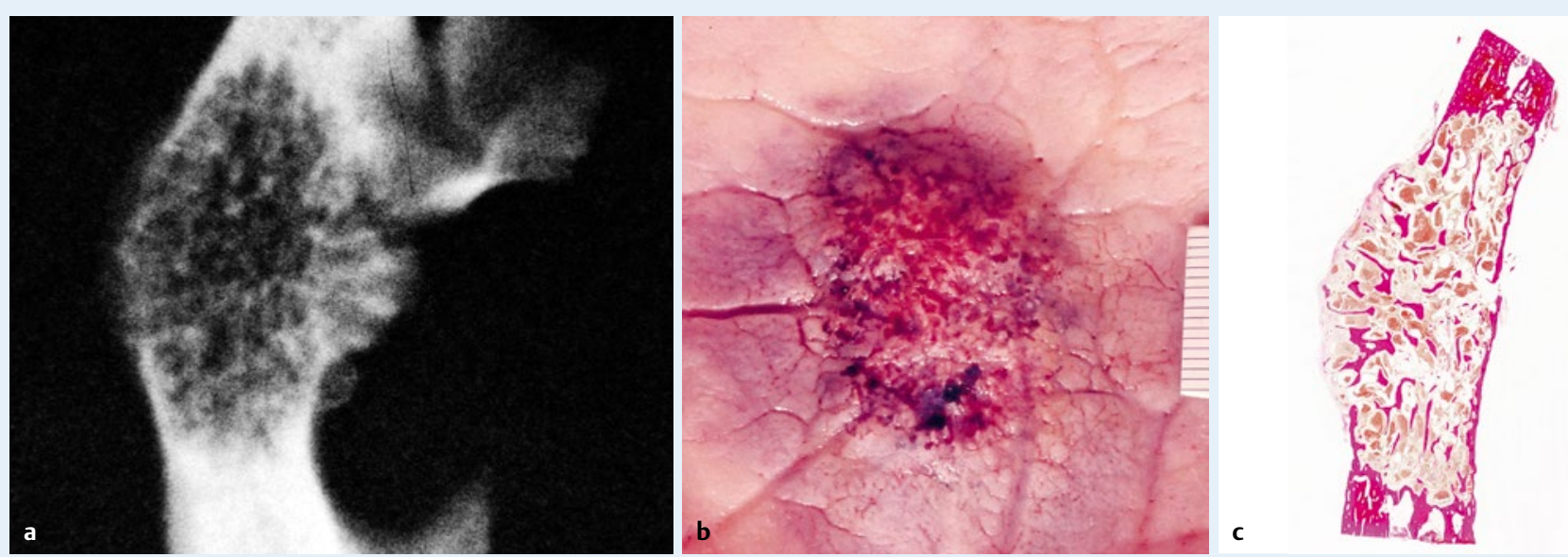

- Abb. 1 Venöse Malformation der Kalotte. Dünnwandige, irreguläre venöse vaskuläre Räume (Erythrozyten-gefüllt) durchsetzen Spongiosa, Kortikalis und führen zu einer charakteristischen periostalen Knochenneubildung. Die vaskulären Räume zeigen keine lobuläre Architektur.
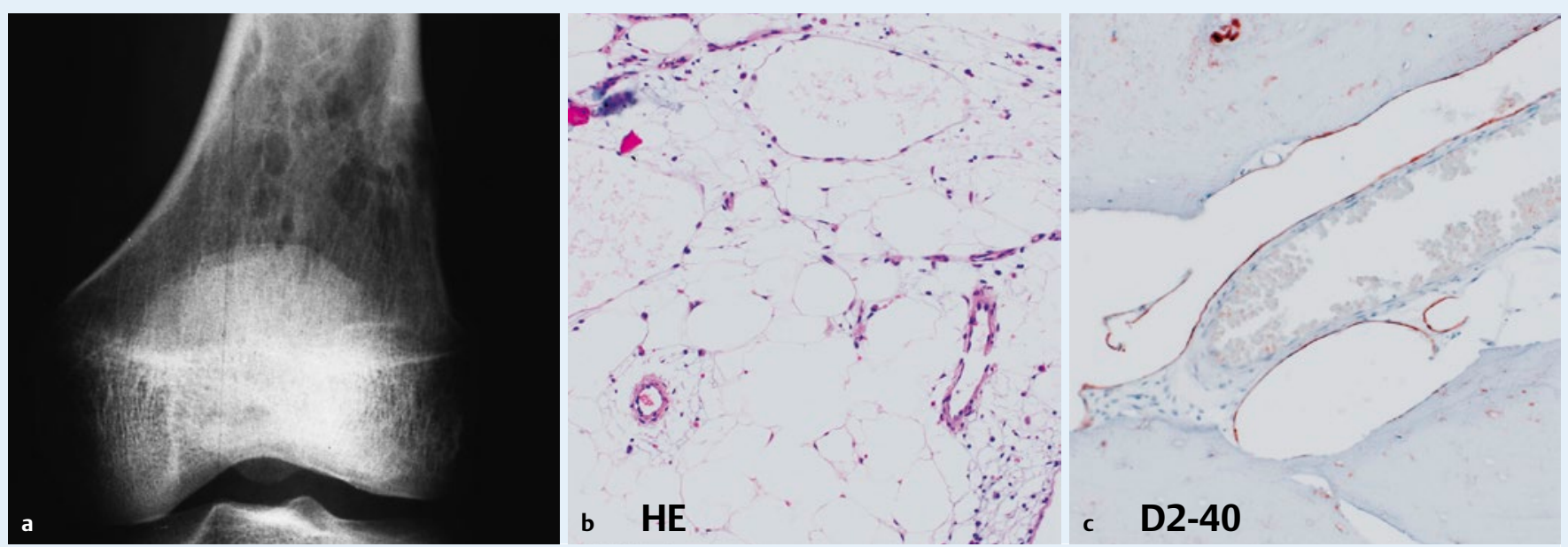

- Abb. 2 Lymphatische Malformation des Femurs. Konventionell-radiologisch konfluierende Osteolysen der distalen Femurmetaphyse mit geringer Randsklerose. Histologisch äußerst dünnwandige vaskuläre Kanäle, ausgekleidet von sehr flachem, blandem Endothel mit Expression von D2-40/ Podoplanin. Das Endothel der zentral gelegenen, mit Erythrozyten gefüllten dünnwandigen Vene ist negativ. 
mit dem radiologisch respektive Dopplersonographisch erkennbaren Flow-Verhalten korreliert werden. Während die meisten vaskulären Malformationen sporadisch auftreten, gibt es jedoch einige typisch familiär gehäuft vorkommende Formen. Sie können isoliert oder kombiniert auftreten und erst im Laufe des Lebens manifest werden (Stiegler 2014).

Venöse Malformationen des Skeletts sind am häufigsten kraniofazial lokalisiert, gefolgt von Wirbelkörpern, Extremitäten und Becken. Radiologisch gehen sie kranial mit einer Expansion und typischen reaktiven Knochenneubildung einher ( $\mathbf{A b b} \mathbf{b} \mathbf{1} \mathbf{a})$, während im Wirbelkörper bei erhaltener Knochenkontur grobe strähnige Sklerosezonen eine Osteolyse begleiten. Die Makroskopie zeigt irregulär angeordnete, dünnwandige, blutgefüllte und daher dunkelrot bis braun imponierende Gefäßräume ( $\mathbf{A b b}$. 1b). Eine lobuläre Architektur ist nicht zu sehen. Histologisch werden diese Gefäßräume durch unregelmäßig konfigurierte glattmuskuläre Gefäßwände begrenzt, überkleidet von flachem Endothel ohne wesentliche Proliferationszeichen ( Abb. 1c). Es können ausgedehnte Thrombosen und reaktive endotheliale Hyperplasien („Masson“) entstehen, die aber keine nukleären Atypien aufweisen und sich so von Angiosarkomen unterscheiden lassen.

Ossäre lymphatische Malformationen betreffen meist das kraniale Skelett und die Extremitäten. Radiologisch sind sie durch konfluierende Osteolysen mit zarter Randsklerose charakterisiert ( $\mathbf{A b b}$. 2a). Makroskopisch finden sich von heller Flüssigkeit gefüllte Räume. Histologisch erkennt man sehr dünnwandige, anastomosierende Kanäle, ausgekleidet durch meist flache, teils etwas vorgewölbte Endothelien mit unauffälligen Kernen ( $\triangleright$ Abb. 2b). Diese lymphothelial differenzierten Endothelien lassen sich seit einiger Zeit immunhistochemisch durch die Expression des onkofetalen Antigens D2-40 (Podoplanin) von hämangiovaskulären Endothelien unterscheiden. Die Gefäßwand zeigt in der Regel kaum eine glattmuskuläre Ausstattung ( $\mathbf{A b b}$. 2c).

Von besonderer Bedeutung sind multifokale oder sogar generalisierte, teils sehr ausgedehnte lymphatische Malformationen, die meist in Wirbelsäule, Thorax oder Schul- tergürtel beobachtet werden. Kommt es zu aberranter Aktivierung von Osteoklasten, erfolgt eine massive Osteolyse, wie sie unter dem Namen „Massive Osteolysis“ von Gorham und Stout beschrieben wurde (Gorham \& Stout 1955) und auch als „Vanishing Bone“ Disease bezeichnet wird. Histologisch konfluieren die lymphatischen Räume und zeigen im Zentrum der involvierten Region lediglich Reste kompakten kollagenen Bindegewebes ohne Knochentrabekel. Vor kurzem konnte die Osteoklastenaktivierung im Mausmodell der Bildung von Makrophagen Kolonie-Stimulierendem Faktor M-CSF zugeschrieben werden (Wang et al. 2017).

\section{Vaskuläre Tumoren}

Die ISSVA Klassifikation unterscheidet gutartige von lokal aggressiven und bösartigen vaskulären Tumoren.

Der häufigste gutartige vaskuläre Tumor ist das infantile Hämangiom, das sich durch drei Wachstumsphasen auszeichnet. Es tritt typischerweise im Bereich der Haut der Kopf-Halsregion auf und manifestiert sich nur ausnahmsweise viszeral. Das ossäre Skelett wird selten sekundär mitbeteiligt, primäre ossäre infantile Hämangiome sind bisher nicht beschrieben. Histologisch ist der lobulär aufgebaute Tumor charakterisiert durch eine kräftige endotheliale immunhistochemische Expression des GLUT1 Glukosetransporters.

Spindelzellhämangiom und kongenitales Hämangiom sind bisher nicht primär intraossär beschrieben worden.

Skelettal tritt hingegen das epitheloide Hämangiom auf, vor allem im Bereich der unteren Extremität und der Kopf-Hals Region. Radiologisch finden sich solitäre oder multifokale exzentrische geographische Osteolysen mit minimaler Randsklerose, kortikaler Erosion und Expansion. Der Tumor kann sich in die angrenzenden Weichteile ausbreiten. Makroskopisch sieht man eine meist umschriebene, solide braun-rote Raumforderung. Die Histologie zeigt eine lobuläre Architektur, teils mit soliden Anteilen. Das Gefäßlumen wird ausgekleidet von zytoplasmareichen, kopfsteinpflasterartigen Endothelien mit einzelnen intrazytoplasmatischen Vakuolen ohne wesentliche Kernpolymorphie ( $>$ Abb. 3a und $\triangleright$ Abb. 3b).
Unter die intermediären, lokal aggressiven Tumoren wird das kaposiforme Hämangioendotheliom eingeordnet, das typischerweise im Weichteilgewebe vorkommt und im Bereich des Skeletts sehr selten ist (Bruder 2009). Radiologisch findet sich eine irreguläre Osteolyse und eine (definitionsgemäß) kleinherdige periossäre Ausdehnung. Die Histologie zeigt konfluierende Noduli von Spindelzellen mit schlitzförmigen Pseudolumina und Erythrozytenextravasaten. Fokal sind auch plumpere, rundliche Zellen mit größeren Kernen und klarem Zytoplasma enthalten. Immunhistochemisch wird fokal der lymphotheliale Marker D2-40 exprimiert. Interessanterweise finden sich Mikrothromben, Hämosiderin und hyaline Globuli. Kaposiforme Hämangioendotheliome (wie auch das verwandte, überwiegend im Kindesalter und in den Weichteilen vorkommende „Tufted Haemangioma“) gehen häufig mit einer Kasabach-Merritt Verbrauchskoagulopathie einher.

Ein weiterer Vertreter lokal aggressiver Gefäßtumoren ist das pseudomyogene (früher auch: epithelioid-sarcoma like) Hämangioendotheliom, das (häufiger) in den Weichteilen und (seltener) im Knochen vorkommen kann und oft multifokal auftritt (Inyang 2016). Es bildet keine Gefäßlumina und zeigt oft große, rundliche bis spindelige Tumorzellen mit breitem, eosinophilem Zytoplasma ( $>$ Abb. 4a und $\triangleright$ Abb. 4c). Immunhistochemisch reagieren die Tumorzellen positiv für Gefäßmarker (CD31, ERG, Fli1) mit Ausnahme von CD34, das konstant negativ ist, sowie Zytokeratinen (DD: Karzinommetastase oder epitheloide Sarkome). Molekularpathologisch zeichnen sich diese Tumoren durch eine FOSB-Translokation aus, welche zu einer Überexpression des Proteins FOSB führt, das immunhistochemisch als Surrogatmarker nachgewiesen werden kann ( $\triangleright$ Abb. 4b und $\triangleright$ Abb. 4d).

Zu den malignen ossären vaskulären Tumoren wird das epitheloide Hämangioendotheliom gerechnet, das sich im Gegensatz zum epitheloiden Hämangiom radiologisch durch eine teils permeative Osteolyse und histologisch durch einen Verlust der lobulären Architektur mit charakteristischer interstitieller, teils prominent myxoider Matrix auszeichnet ( $\mathbf{A} \mathbf{A b b} . \mathbf{3} \mathbf{c}$ und $\boldsymbol{A}$ Abb. $\mathbf{3 d}$ ). Die Zellkerne sind mäßig pleomorph und zeigen prominente Nukleolen, sowie einzelne typi- 

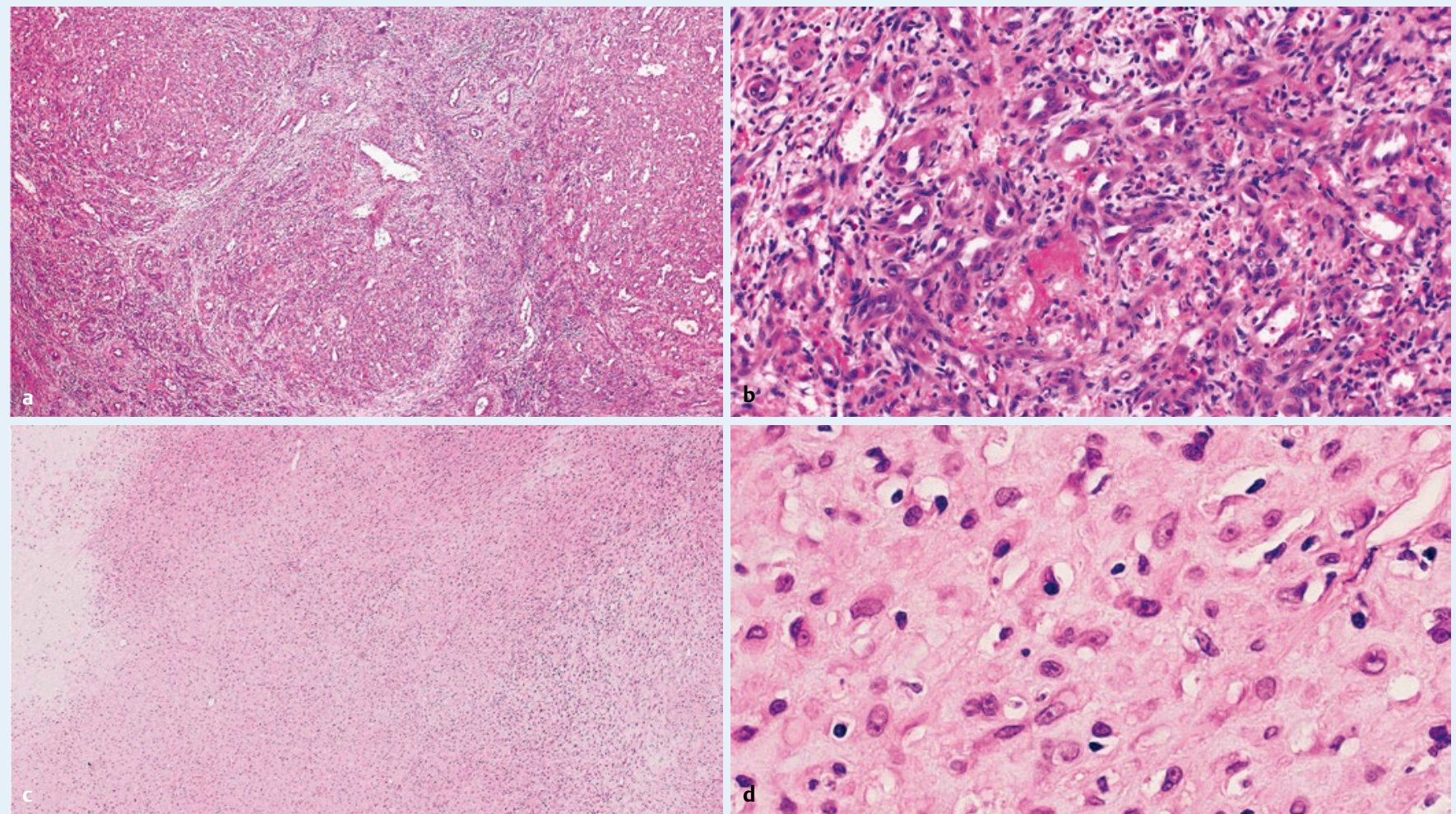

>Abb. 3 Epitheloides Hämangiom und Epitheloides Hämangioendotheliom. Das Epitheloide Hämangiom (A-B) zeigt histologisch eine erhaltene lobuläre Architektur mit Ausbildung intakter Gefäßstrukturen, während beim Epitheloiden Hämangioendotheliom (C-D) der lobuläre Aufbau verloren geht. Die Tumorzellen des epitheloiden Hämangioendotheliomes liegen in einer charakteristischen, teils myxoiden, teils kollagenisierten interstitiellen Matrix und bilden zytoplasmatische Vakuolen aus.
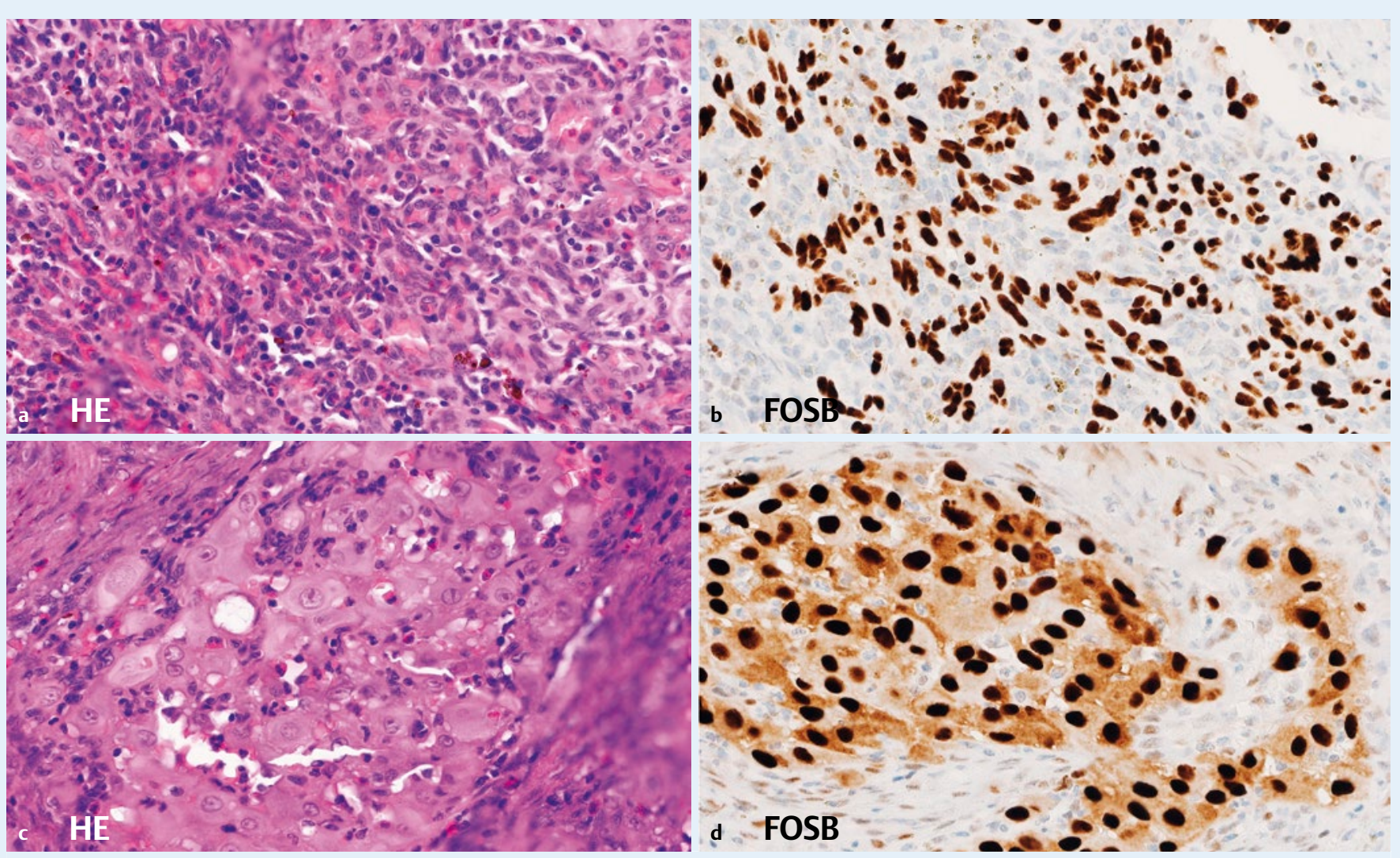

Abb. 4 Pseudomyogenes Hämangioendotheliom. Ausgedehnt hämorrhagisch-nekrotische Tumormasse mit kleinen Herden teils spindelzelliger (a), teils epithelioid (c) imponierender, zytoplasmareicher Tumorzellaggregate mit kräftiger nukleärer Expression von FOSB (b und d). 
sche Mitosefiguren. Seit 2001 ist bei einem Teil der epitheloiden Hämangioendotheliome eine rekurrente, diagnostisch nutzbare Translokation bekannt, der ein CAMTA1 Rearrangement zugeordnet werden konnte (Mendlick 2001, Patel 2015).

Angiosarkome können monostotisch oder multifokal auftreten und betreffen vorwiegend die langen Röhrenknochen. Radiologisch sind sie durch unregelmäßig begrenzte Osteolysen ohne Randsklerose charakterisiert ( $\triangleright$ Abb. 5 a). Histologisch zeigen sie ein vielfältiges Bild. Eine Vasoformation fehlt, anastomosierende kanalartige Strukturen, die von plumpen Tumorzellen ausgekleidet sind, werden jedoch oft gebildet und können den Verdacht in Richtung eines Gefäßtumors lenken. Außerdem bilden die Tumorzellen solide Aggregate und Rasen mit infiltrativem Wachstumsmuster und ausgedehnter Weichteilkomponente. Zytoplasmatische Vakuolen sind selten. Es findet sich eine ausgeprägte Kernpolymorphie und Hyperchromasie mit zahlreichen auch atypischen Mitosefiguren ( $>$ Abb. $\mathbf{5 b}$ ). Das Zytoplasma kann eine epitheloide oder spindelige Konfiguration annehmen. Da eine Zytokeratinexpression vorkommt ( $\triangleright \mathbf{A b b}$. $5 \mathbf{b}$ Inset), ist die Verwechslung mit Karzinommetastasen möglich, wenn nicht zusätzlich endothel-typische Antikörper wie ERG, CD31 oder Faktor VIII eingesetzt werden. Auf der molekularen Ebene werden bei Angiosarkomen Mutationen im KDR Gen beschrieben sowie MYC Amplifikationen bei Strahlen-Assoziation (Mulliken 2013).

\section{Pathomorphologische und Genetische Diagnostik}

Das pathomorphologische diagnostische Instrumentarium konnte in den letzten Jahren um ein Spektrum von immunhistochemisch anwendbaren Antikörpern erweitert werden (siehe - Tab. 2). Ein „Angio-Panel“ von CD31, D2-40, SMA, GLUT1, MIB1 und WT1 hat sich über die Jahre in unserer Institution als nützlich erwiesen. WT1 wird unserer Erfahrung nach vor allem in den Membranen und im Zytoplasma der Endothelien von gegenwärtig als vaskulären Tumoren aufgefassten Läsionen wie z. B. einem epitheloidem Hämangiom exprimiert, wohingegen Endothelien z. B. von venösen und lymphatischen Malformationen negativ bleiben.
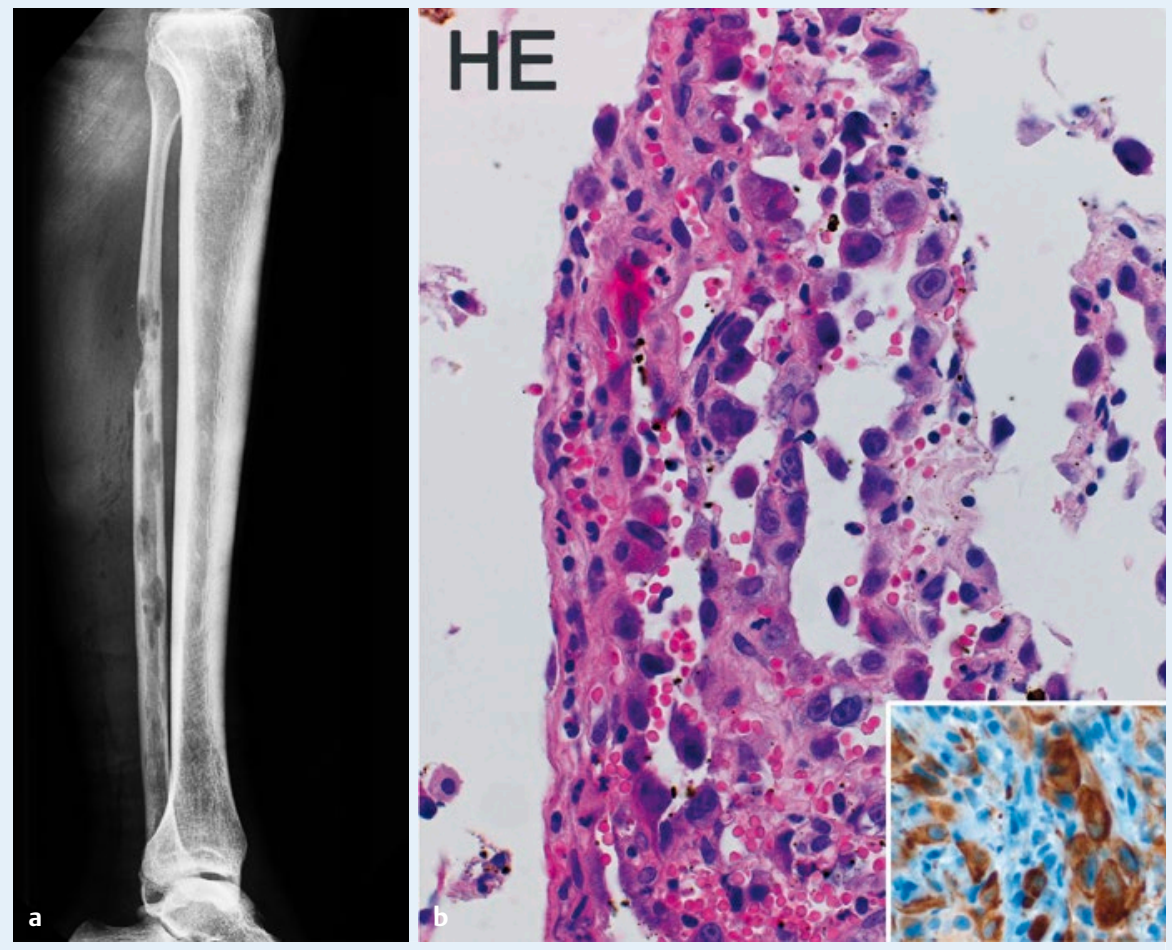

Abb. 5 Angiosarkom der Fibula. Ausgedehnte Osteolysen mit Destruktion der Kortikalis und Weichteilinfiltration, histologisch ungeordnete Rasen maligner Zellen ohne erkennbare Ausbildung von Gefäßstrukturen. Immunhistochemisch können die Tumorzellen mit epithelialen Markern (Zytokeratin- Inset) reagieren: DD Karzinommetastase.

> Tab. 2 Immunhistochemisches Angio-Panel zur Routinediagnostik bei vaskulären Läsionen (Auszug).

\begin{tabular}{|c|l|l|}
\hline Antikörper & $\begin{array}{l}\text { Expressions- } \\
\text { muster }\end{array}$ & Diagnostische Bedeutung \\
\hline CD31 & Membranär & $\begin{array}{l}\text { Vaskuläre und lymphatische Endothelien bei allen vaskulären } \\
\text { Malformationen und Tumoren }\end{array}$ \\
\hline D2-40 & Membranär & Lymphatische Endothelien/ Lymphothel \\
\hline SMA & Zytoplasmatisch & $\begin{array}{l}\text { Glatte Muskulatur, perivaskuläre Zellen: Darstellung der (fast } \\
\text { immer) alterierten Wandarchitektur, z. B. bei kapillären Häman- } \\
\text { giomen und venösen Malformationen }\end{array}$ \\
\hline GLUT1 & $\begin{array}{l}\text { Membranär und } \\
\text { zytoplasmatisch }\end{array}$ & $\begin{array}{l}\text { Expression bei infantilen Hämangiomen. Bei anderen vaskulären } \\
\text { Läsionen äußerst selten (z. B. ein Teil der Angiosarkome) }\end{array}$ \\
\hline WT1 & Membranär und & $\begin{array}{l}\text { Expression in Endothelien von Tumoren, sowie in normalen } \\
\text { Kapillaren und normalen arteriellen Endothelien. In den Endo- } \\
\text { thelien z. B. von venösen Malformationen negativ. }\end{array}$ \\
\hline MIB1 & Nukleär & $\begin{array}{l}\text { Sehr geringe Expression/ Proliferationsrate (<1\%) in Endothelien } \\
\text { von vaskulären Malformationen (ohne reaktive Veränderungen). } \\
\text { Höhere Expression/ Proliferationsrate (ca. 10-30\%) in Häman- } \\
\text { giomen. }\end{array}$ \\
\hline CAMTA1 & Nukleär & $\begin{array}{l}\text { Korrelat einer CAMTA1 Fusion beim Epitheloiden Hämangioen- } \\
\text { dotheliom. }\end{array}$ \\
\hline FOSB & Nukleär & $\begin{array}{l}\text { Korrelat einer FOSB Fusion beim Pseudomyogenen Hämangio- } \\
\text { endotheliom }\end{array}$ \\
\hline
\end{tabular}

Aktuell ergänzen Antikörper wie CAMTA1 und FOSB das Panel und lassen die nukleärelat einer Translokation am histologischen re Überexpression dieser Proteine als Kor- 
Für eine Vielzahl dieser vaskulären Läsionen sind durch die modernen molekularen Methoden (NGS, Exomanalysen ect. und Familienuntersuchungen) kausale Gene ( $\triangleright$ Tab. 3) bekannt und stehen in der genetischen Routinediagnostik zur Verfügung. Neben der Diagnosestützung, Nachweis einer hereditären Disposition, Möglichkeiten diagnostischer und prädiktiver Testungen in den Familien sowie pränataler Diagnostik gewinnt die Detektion von pathogenen Sequenzveränderungen auch im therapeutischen Ansatz immer größere Bedeutung, z. B. Vermeidung wachstumsfördernder Medikamente beim Proteus-Syndrom (Biesecker 2018).

Von besonderer Relevanz ist die Differenzierung in somatische und Keimbahnspezifische Sequenzveränderungen. Keimbahnmutationen sind mit einer hereditären familiären Disposition verbunden. Ihr Nachweis sollte eine weitere genetische Diagnostik bei weiteren Familienangehörigen nach sich ziehen. Somatische Genveränderungen z. B. das CAMTA1-Rearrangement beim epitheloiden Hämangioendotheliom, entstehen postzygotisch in den aberranten Zellen und sind im histopathologischen Kontext in der Differentialdiagnostik der Läsion von großer Bedeutung. Sie werden nicht vererbt. Ein Hinweis auf eine Keimbahnveränderung ergibt sich dann, wenn im untersuchten Tumorgewebe bei einer autosomal dominanten Disposition biallelische Veränderungen (beide Genkopien zeigen Mutationen) gefunden werden. Es ist dann von einer heterozygoten Keimbahnveränderung und einer zusätzlichen somatischen Mutation auszugehen, welche einer nachfolgenden humangenetischen Analyse bedürfen z. B. mittels einer Blutprobe des Patienten. Aber auch Mosaike auf molekularer Ebene sind bekannt, deren Nachweis nur bei klinisch begründetem Verdacht zur Diagnosesicherung sinnvoll ist, z. B. Proteus - Syndrom (OMIM 176920) mit AKT1- Mutationen (postzygotisches Mosaik).

Die klinische Variabilität von Keimbahnmutationen ergibt sich aus der Penetranz (= ob ein bestimmtes Merkmal vorhanden ist) und variablen Expressivität (= in welchem Ausmaß dieses Merkmal vorhanden ist) der Gene sowie dem Einfluss assoziierter Veränderungen ( $\triangleright$ Abb. 6).

-Tab. 3 Genveränderungen vaskulärer Läsionen.

\begin{tabular}{|l|l|}
\hline Vaskuläre Läsion & Assoziierte Genveränderungen \\
\hline Kapillare Malformation & GNAQ, RASA1, ENG, ACVR1 \\
\hline Lymphatische Malformationen & $\begin{array}{l}\text { FLT4/VEGRFR3, VEGFC, GJCI2, FOXC2, SOX18, GATA2, CCBE1, } \\
\text { KIF11, PTEN14 }\end{array}$ \\
\hline Venöse Malformationen & $\begin{array}{l}\text { TIE2 somatic, TIE2, Glomulin } \\
\text { KRIT1, Malcavermin, PDCD10 }\end{array}$ \\
\hline Arteriovenöse Malformationen & ENG, ACVRL1, SMADA4, RASA1 \\
\hline $\begin{array}{l}\text { Syndromassoziierte venöse } \\
\text { Malformationen }\end{array}$ & RASA1, GNAQ, PIK3CA, PTEN, STAMBP, AKT1, \\
\hline
\end{tabular}
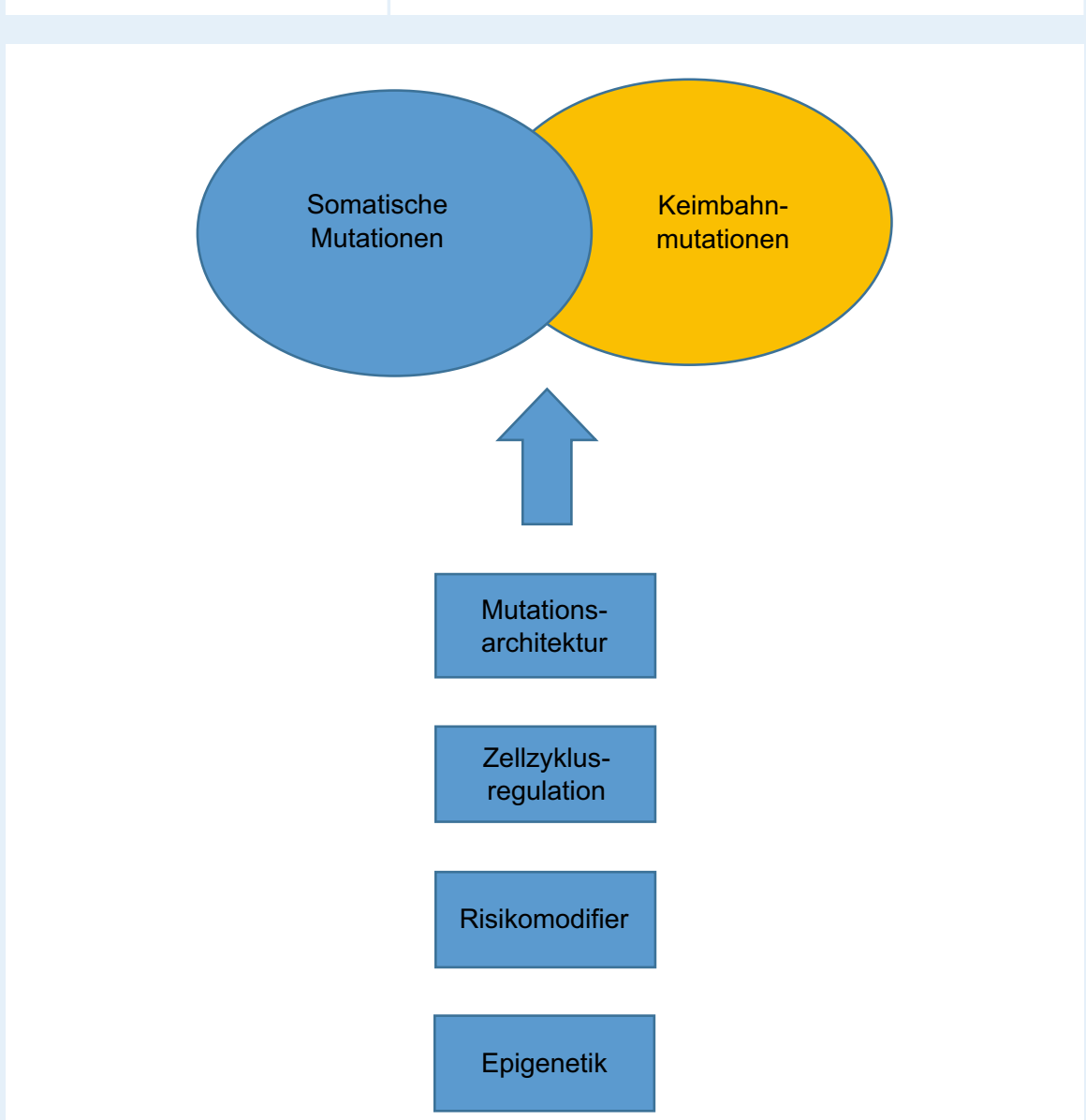

Abb. 6 Genetische Architektur. Relevante Einflüsse bezüglich Penetranz und Expressivität auf die genetische Architektur somatischer Mutationen und Keimbahnmutationen.

Genetisch bedingte komplexe vaskuläre Anomalien mit assoziierten Veränderungen stellen eine besondere Gruppe der vaskulären Läsionen dar. Diese Erkrankungen können mit anderen benignen oder malignen Tumoren z. B. Adenomen der Nebenschilddrüse, Skelett- und Augenveränderungen assoziiert sein. Bei diesen Anomalien sind neben dem Nachweis von Mutationen in der konstitutiven DNA auch somatische Mosaike beschrieben. Zur Diagnose führt die spe- zifische Kombination der klinischen Symptomatik und die Familienanamnese.

\section{Zusammenfassung}

Vaskuläre Läsionen stellen auch im Skelett ein komplexes Spektrum dar. Fortschreitende molekulare Erkenntnisse haben gegenwärtig eine binäre Klassifikation ermöglicht, in der strukturelle Malformationen proliferativen Tumoren gegenübergestellt werden. 
Die Patienten sollten sowohl diagnostisch als auch therapeutisch in spezialisierten interdisziplinären Zentren betreut werden. Bei Hinweisen auf eine mögliche hereditäre Disposition (klinische Symptomatik, gewebsspezifische histopathologische und molekular-pathologische Befunde oder die Familiengeschichte) sollte immer die Möglichkeit einer genetischen Abklärung im Rahmen einer humangenetischen Beratung erwogen werden.

\section{Autorinnen/Autoren}

Dipl. Med. Dorothea Wand

Institut für Medizinische Genetik, Universitätsspital Basel

\section{Prof. Dr. med. Gernot Jundt}

Institut für Pathologie

Prof. Dr. med. Elisabeth Bruder

Institut für Pathologie

\section{Korrespondenzadresse}

Prof. Dr. med. Elisabeth Bruder Institut für Medizinische Genetik und Pathologie Universitätsspital Basel Schönbeinstrasse 40 4031 Basel - Schweiz Tel. + 41611652757 Fax +41612653194 E-Mail: elisabeth.bruder@unibas.ch
[1] Bruder E et al. Vascular lesions of bone in children, adolescents and young adults. A clinicopathologic reappraisal and application of the ISSVA classification. Virchows Archiv 2009; 454: 161-179

[2] Biesecker LG, Sapp JC. Proteus Syndrome. 2012 Aug 9 [Updated 2019 Jan 10]. In: Adam MP, Ardinger HH, Pagon RA, et al., editors. GeneReviews ${ }^{\circledR}$ [Internet]. Seattle (WA): University of Washington, Seattle; 1993-2019. Available from: https://www. ncbi.nlm.nih.gov/books/NBK99495/

[3] Dabska M. Malignant endovascular papillary angioendothelioma of the skin in childhood. Clinicopathologic study of 6 cases. Cancer 1969; 24: 503-510

[4] Duffy K. Genetics and syndromes associated with vascular malformations; Pediatr Clin North Am 2010; 57(5): 1111-1120

[5] Eivazi B, Werner JA. Venöse und arteriovenöse Malformationen im Kopf-Hals-Bereich. Therapeutische Möglichkeiten und Herausforderungen. In: HNO, Springer, Berlin Heidelberg 2014: 19-24

[6] Fanburg-Smith J et al. Papillary intralymphatic angioendothelioma (PILA): a report of twelve cases of a distinctive vascular tumor with phenotypic features of lymphatic vessels. Am J Surg Pathol 1999; 23: 1004-1010

[7] Freyschmidt J, Ostertag H, Jundt G. Knochentumoren mit Kiefertumoren. Klinik, Radiologie, Pathologie. 3. Aufl. Springer Verlag Heidelberg 2010

[8] Gorham LW, Stout AP. Massive osteolysis (acute spontaneous absorption of bone, phantom bone, disappearing bone); its relation to hemangiomatosis. J Bone Joint Surg Am 1955; 37-A: 985-1004

[9] Inyang A et al. Primary Pseudomyogenic Hemangioendothelioma of Bone. Am J Surg Pathol 2016 ;40:587-598

[10] ISSVA Classification of Vascular Anomalies (C)2014 International Society for the Study of Vascular Anomalies Available at „issva.org/ classification“ Accessed [February 5th, 2018]

[11] Mendlick MR et al. Translocation $t(1 ; 3)$ (p36.3;q25) Is a Nonrandom Aberration in Epithelioid Hemangioendothelioma. Am ] Surg Pathol 2001; 25: 684-687

[12] Mulliken JB, Burrows PE, Fishman S]. Mulliken \& Young's Vascular Anomalies. Hemangiomas and Malformations. 2nd edition, Oxford University Press, New York 2013

[13] Patel NR et al.: Molecular characterization of epithelioid haemangioendotheliomas identifies novel WWTR1-CAMTA1 fusion variants. Histopathology 2015; 67: 699-708

[14] Stiegler H, Wohlgemuth WA, Saleh A. A vaskuläre Malformationen - klinische Bilder: Stellenwert der Duplexsonographie. In: Gefässchirurgie, Springer, Berlin Heidelberg 2014: 302-304

[15] Wang W et al. Lymphatic Endothelial Cells Produce M-CSF, Causing Massive Bone Loss in Mice. J Bone Miner Res 2017; 32: 939-950 